\title{
Cryptosporidiosis in a patient with ankylosing spondylitis treated with adalimumab
}

\author{
Fernando Augusto Chiuchetta ${ }^{1}$
}

\begin{abstract}
Cryptosporidiosis is a parasitic disease caused by a protozoan called Cryptosporidium sp. An increased number of diagnoses were made in the last 20 years, especially in patients with immunodeficiency like the acquired human immunodeficiency syndrome and induced immunodeficiency, such as in transplant patients and those who need frequent hemodialysis, has been observed. We report the case of a young patient with ankylosing spondylitis treated with adalimumab who developed chronic diarrhea secondary to cryptosporidiosis
\end{abstract}

Keywords: ankylosing spondylitis, adalimumab, cryptosporidiosis.

\section{INTRODUCTION}

Cryptosporidiosis is a parasitic disease caused by the protozoa Cryptosporidium sp, isolated by Tyzzer in 1907. Since the decade of 1980, it has become clinically important due to the development of difficult-to-control chronic diarrhea in patients with immunodeficiency, such as acquired human immunodeficiency syndrome, as well as immunodeficiencies induced in transplant patients and in those who need frequent hemodialysis. ${ }^{1}$

Currently, evidence indicates that biological agents against tumor necrosis factor (anti-TNF) represent a good therapeutic option in patients with severe a disease ctivity of ankylosing spondylitis. ${ }^{2}$ As patients treated with biological agents are at higher risk of developing infections, adequate measures to prevent, rapidly identify, and treat these severe infections should be applied.

\section{CASE REPORT}

This is a report of a 33 years old male patient with ankylosing spondylitis diagnosed in 2002 (inflammatory lumbalgia, HLA-B27 positive, and bilateral sacroiliitis). He was initially treated, with meloxicam $(15 \mathrm{mg} /$ day $)$ and methotrexate $(10 \mathrm{mg} /$ week $)$ with clinical improvement. In May 2007, the patient presented worsening pain, knees edema, and difficulty walking. The dose of methotrexate was increased to $20 \mathrm{mg} /$ week, without clinical response. Sulfasalazine $(2,000 \mathrm{mg} /$ day $)$ and prednisone $(10 \mathrm{mg} /$ day $)$ were added to the therapeutic regimen. In September 2007, the administration of adalimumab was indicated due to persistent axial pain and peripheral arthritis with BASDAI of 7.0. Sulfasalazine was discontinued. The patient showed progressive improvement of pain and, consequently, the dose of the other drugs was gradually reduced. In April 2008, the patient was taking adalimumab (40 mg every two weeks), methotrexate $(10 \mathrm{mg} /$ week $)$, prednisone $(5 \mathrm{mg} /$ day $)$ and nimesulide (100 mg/day) with BASDAI of 2. In May 2008, the patient developed liquid diarrhea without blood, with six episodes a day accompanied by nausea, asthenia, fever, and weight loss. He was seen by a gastroenterologist who requested abdominal ultrasound, which was normal, and HIV was negative. Stool parasitological test showed the presence of a large amount of yeasts, by the Faust and Hoffman method, and the presence of Cryptosporidium oocysts was demonstrated by the Ziehl Nielsen method, which showed the presence of Cryptosporidium oocysts. He was referred to

Received on 07/01/2009. Approved on 04/20/2010. We declare no conflict of interest.

Hospital XV - Curitiba, PR, Brazil

1. Former Rheumatology Resident of UFPR, Rheumatologist by the SBR, and Rheumatology Counselor for the Orthopedics Residency at Hospital XV - Curitiba, PR, Brazil

Correspondence to: Dr. Fernando Augusto Chiuchetta. Reumatologia para residência de ortopedia do Hospital da XV. Rua Alberto Foloni, 1093.

CEP: 80540-000, Curitiba,PR, Brazil.E-mail: Fmch8@uol.com.br 
an infectologist who instituted treatment with nitazoxanide, $1,000 \mathrm{mg} /$ day for 14 days, with improvement of the diarrhea and the stool parasitologic became negative. Adalimumab was discontinued due to the presence of cryptosporidiosis and herpes zoster in the thorax, which the patient developed one month after the diarrhea onset.

\section{DISCUSSION}

Cryptosporidium parvum (C. parvum) is a protozoon belonging to the order eucoccidia, family cryptosporidiidae. Oocysts, which are eliminated in the feces of the host, are the infecting form. ${ }^{3}$ Other apicomplexa pathogens include the parasite plasmodium (malaria) and toxoplasma (toxoplasmosis). Cryptosporidium is a non-specific parasite, i.e., it satisfactory develops in different types of hosts, such as bovine, swine, birds, mice, and also in men. It has a monoxenic cycle, requiring only one host to survive. ${ }^{4}$ It is transmitted, most often, by water contaminated with oocysts, which is ingested, or in pools, parks, and bathtubs. Sexual transmission, both heterosexual and homosexual, is another important means of transmission of cryptosporidiosis. This type of transmission is possible because it is a protozoonosis with fecal-oral transmission. Foods, such as raw meats, raw and poorly washed vegetables, as well as non-pasteurized milk, represent less common, but equally important, means of transmission; air-borne transmission also occurs, being responsible for respiratory cryptosporidiosis. ${ }^{3}$ The severity of this enteritis is variable, and it can evolve from a relatively oligoasymptomatic type, with a few semi-soft evacuations a day, to a choleriform type, characterized by severe aqueous diarrhea ( 1 to 20 liters a day), diarrhea, without pus or blood, along with severe abdominal pain, marked weight loss, anorexia, and malaise. Nausea, vomiting, malabsorption syndrome, fever, and myalgia can also be present. Approximately 10 to $15 \%$ of patients may present associated cholangitis or cholecystitis. Rarely, Cryptosporidium can cause interstitial pneumonitis similar to pneumocystosis. ${ }^{5}$ Cryptosporidiosis has an incubation period of 2 to 14 days.

Diagnosis is attained by analyzing at least three stool samples or by the identification of the parasite in bowel biopsy. Several diagnostic techniques have been described, such as concentration, staining, and microscopic analysis, immunologic methods, and molecular techniques. Polymerase chain reaction (PCR) has been improved after the genome of C. parvum was sequenced; it has a high specificity and can detect small amounts of the parasite in human and bovine feces or in paraffin-fixed biopsies. For microscopic analysis, the staining methods recommended include modified ZiehlNeelsen (ZNm) and acid-fast-trichrome (AFT). Although AFT has a lower cost, the ZNm method is considered the most adequate, with $100 \%$ sensitivity and specificity for C. parvum. ${ }^{6}$ Several drugs have been tested and used in the treatment of cryptosporidiosis since the decade of 1980 , without satisfactory response regarding the cure, both clinical and parasitological. ${ }^{7}$ Clinical response and reduction in the parasitic load with macrolides (azithromycin, spiramycin, and roxithromycin) and paromomycin have been reported. ${ }^{8}$ In a recent study of patients with Cryptosporidium-induced diarrhea, nitazoxanide significantly reduced the duration of diarrhea and other gastrointestinal symptoms when compared to placebo. ${ }^{9}$ Antispasmodic, antiediarrhea, and anti-emetic drugs can be prescribed, and vigorous hydration, oral or parenteral, is suggested when necessary. Enteral or parenteral nutrition can be indicated in more severe cases, usually presenting more severe malnutrition.

Severe infections represent an important concern in patients with rheumatic diseases, being among the most important causes of mortality in rheumatoid arthritis and other inflammatory rheumatic diseases. The underlying disease, its the degree of activity, degree of disability, as well as the presence of comorbidities, besides treatment with immunosuppressors, are independent risk factors. Tumor necrosis factor has a fundamental role in defending the organism against bacterial and viral invasion by recruiting neutrophils, eosinophils, and macrophages to the site of infection. ${ }^{10}$ All those factors are strictly interconnected. In a recent metanalysis of nine randomized clinical trials of infliximab or adalimumab in rheumatoid arthritis, an increase in the risk of severe infections in patients undergoing these treatments was observed when compared to those receiving placebo. ${ }^{11}$ Several case reports have described the association between anti-TNF therapy and the development of opportunistic infection, such as histoplasmosis, listeriosis, coccidioidomycosis, candidiasis, pneumocystosis, and aspergillosis. However, these infections are rare. ${ }^{12}$ Listing et al. compared infection rates in patients treated with biological agents with that of patients treated with conventional therapy. Compared to the control group, the incidence of severe infections was 2.7-2.8 times higher in patients treated with biological agents, and the incidence of adverse events in general was 3.3-4.1 times higher. ${ }^{13}$ The present report alerts for the possibility of an infection (cryptosporidiosis) during treatment with biological agents, in this case adalimumab, which is a rare situation, but of important clinical significance. 


\section{REFERÊNCIAS}

\section{REFERENCES}

1. Cimerman S, Castañeda CG, Iuliano WA, Palacios R. Perfil das entereroparasitoses diagnosticadas em pacientes com infecção pelo vírus HIV na era da terapia anti-retroviral potente em um centro de referência em São Paulo, Brasil. Parasitol Latinam 2002; 57:111-9.

2. Consenso Brasileiro de Espondiloartropatias: Espondilite Anquilosante e Artrite Psoriásica Diagnóstico e Tratamento Primeira Revisão Rev Bras Reumatol 2007; 47(4):233-42.

3. Fayer R, Morgan U, Upton SJ. Epidemiology of Cryptosporidium: transmission, detection and identification. Int J Parasitol 2000; 30:1305-22.

4. Martins CAP, Guerrant RL. Cryptosporidium and Cryptosporidiosis. Parasitol Today 1995; 11(11).

5. Petersen C. Cryptosporidium. AIDSFile 1994; 8(3):1-3.

6. Rigo C, Franco R. Comparision between the modified ZiehlNeelsen and Acid-Fast-Trichome methods for fecal screening of Cryptosporidium parvum and Isospora belli. Rev Soc Bras Med Trop 2002; 35(3):209-14.

7. Feldman M. Sleisenger \& Fordtran's Gastrointestinal and Liver Disease, 7th ed. Saunders, 2002.
8. Soave R. Treatment strategies for criptosporidiosis. Ann NY Acad Sci 1990; 616:442-51.

9. Rossignol JF, Ayoub A, Ayers MS. Treatment of diarrhea caused by Cryptosporidium parvum: a prospective randomized, doubleblind, placebo-controlled study of nitazoxanide. J Infect Dis 2001; 184:103-6.

10. Strangfeld A, Listing J. Bacterial and opportunistic infections during anti-TNF therapy. Best Pract Res Clin Rheumatol 2006; 20(6):1181-95.

11. Bongartz T, Sutton AJ, Sweeting MJ. Anti-TNF antibody therapy in rheumatoid arthritis and the risk of serious infections and malignancies: systematic review and meta-analysis of rare harmful effects in randomized controlled trials. JAMA 2006; 295(19):2275-85.

12. Filler SG, Yeaman MR, Sheppard DC. Tumor necrosis factor inhibition and invasive fungal infections. Clin Infect Dis 2005; 41(3):208-12.

13. Listing J, Strangfeld A, Kary S. Infections in Patients With Rheumatoid Arthritis Treated With Biologic Agents. Arthritis Rheum 2005; 52(11):3403-12. 